\title{
Factors associated with low-level physical activity in elderly patients with chronic obstructive pulmonary disease
}

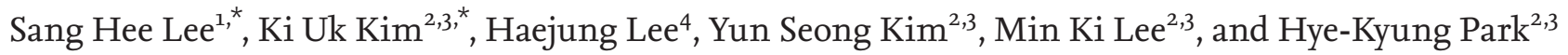

\begin{abstract}
${ }^{1}$ Department of Internal Medicine, Wonkwang University Sanbon Hospital, Gunpo; ${ }^{2}$ Department of Internal Medicine, Pusan National University School of Medicine, Busan; ${ }^{3}$ Medical Research Institute, Pusan National University Hospital, Busan; ${ }^{4}$ Department of Nursing, Pusan National University College of Nursing, Yangsan, Korea
\end{abstract}

Received: March 18, 2016

Revised : July 26, 2016

Accepted: October 26, 2016

\section{Correspondence to}

Hye-Kyung Park, M.D.

Department of Internal Medi-

cine, Pusan National University

School of Medicine, 179 Gudeok-

ro, Seo-gu, Busan 49241, Korea

Tel: $+82-51-240-7802$

Fax: +82-51-254-3127

E-mail: parkhk@pusan.ac.kr

*These authors contributed equally to this work.
Background/Aims: In patients with chronic obstructive pulmonary disease (COPD), the extent of physical activity (PA) is correlated with disease severity and prognosis. However, factors associated with low-level PA in elderly COPD patients are not known. We assessed the levels of PA and clinical factors associated with low-level of PA in elderly COPD patients.

Methods: This was a secondary analysis of a multicenter, prospective study of 245 patients with COPD. Among them, 160 patients with 65 years or more were included. Three PA groups were defined with respect to daily activity time (low, moderate, and high). Health related quality of life (HRQL) was measured using St. George's respiratory questionnaire (SGRQ) and 36-item short-form health survey. Anxiety and depression status were assessed employing the hospital anxiety and depression scale (HADS). Multivariate logistic regression was performed to identify independent predictors of low-level PA in elderly COPD patients.

Results: Of all the 160 patients, 103 (64.4\%) engaged in low-level PA. Upon univariate analysis, a decreased exercise capacity (6-minute walk test $<250 \mathrm{~m})$, an increased dyspnea (the modified medical research council [MMRC] dyspnea scale $\geq 2$ ), a decreased HRQL (total SGRQ score), and a presence of depression (HADS-D $\geq 8$ ) were significantly associated with low-level PA. Upon multivariate analysis, an MMRC grade $\geq 2$ (hazard ratio [HR], 2.550; $p=0.034$ ), and HADS-D $\geq 8$ (HR, 2.076; $p=0.045$ ) were independently associated with low-level PA in elderly COPD patients.

Conclusions: Two-thirds of elderly patients with COPD reported low-level of PA. More severe dyspnea and a presence of depression were independently associated with low-level PA in elderly COPD patients.

Keywords: Pulmonary disease, chronic obstructive; Aged; Dyspnea; Depression

\section{INTRODUCTION}

The numbers of older people continue to grow rapidly worldwide, especially in developing countries [1]. Aging is associated with accumulation of damage to molecules, cells, and tissues, and elderly individuals are often more susceptible to diseases, comorbidities, reductions in quality of life, disabilities, and mortality [2]. However, interindividual differences in the rates of functional organ decline are evident [3]. Chronic obstructive pulmonary disease (COPD) is a major cause of morbidity and mortality in old age [4]. Patients with COPD are impaired 
in terms of all of ventilatory mechanics, pulmonary gas exchange, cardiac function, and other systemic features [5]. Although COPD treatments including bronchodilators, glucocorticoids, and oxygenation increase life expectancy, the interval between the onset of disability and death is thus extended. Elderly patients with COPD are frequently impaired in terms of their social life and functional capacity [6].

The level of physical activity (PA) in patients with COPD is lower than that in healthy age-matched controls [7]. One previous study found that more physically active patients with COPD exhibited better functional status in terms of the diffusing capacity of lung carbon monoxide and expiratory muscle strength as well as lower-level systemic inflammation [8]. In addition, high-level PA reduces both the number of hospital admissions caused by COPD exacerbation as well as the mortality rate [9]. Risk factors associated with low-level PA in patients with COPD have been reported in several studies $[10,11]$. Because most works were cross-sectional in nature, it has not been possible to draw causal inferences from the established associations [12]. In addition, most studies included middle-aged patients, and factors associated with low-level PA in elderly patients with COPD were not emphasized. Therefore, the aims of the present study were to assess PA levels in elderly patients with COPD and identify clinical factors associated with low-level PA in such patients.

\section{METHODS}

\section{Patients and study design}

This was a secondary analysis of data from a multicenter prospective cross-sectional study conducted from March 2010 to November 2010 [13]. In total, 249 patients with COPD of varying severity were recruited from six institutions. Of these, 160 patients aged $\geq 65$ years were included in the present study. Clinically unstable patients (i.e., those requiring changes in medication dose or frequency, exhibiting disease exacerbation, or who had been admitted to hospital in the preceding 6 weeks) were excluded. The study protocol was approved by the Institutional Ethics Committee of Pusan National University Hospital, and written informed consent was obtained from all patients.

\section{Data collection}

The following data were collected from patient records: age, sex, educational level, marital status, smoking status (current-, ex-, or never-smoker), and cumulative smoking history (pack-years). Body mass index was calculated as weight in kilograms divided by height in meters squared. All patients underwent lung function testing and completed a questionnaire. In terms of PA, the frequency and duration of walking or other exercise, such as bicycling, gardening, stretching, aerobics, dancing, climbing, swimming, soccer, tennis, and jogging, were explored. Patients who exercised for $<30$ minutes per day comprised the low-level PA group; the high-level PA group comprised those who exercised for $\geq 60$ minutes per day.

The severity of dyspnea was assessed using the modified medical research council (MMRC) scale. Patients with MMRC scores of $\geq 2$ were regarded as having more severe symptoms. The 6 -minute walk test was performed (the total distance walked over 6 minutes was recorded). Health related quality of life (HRQL) was measured using the St. George's respiratory questionnaire (SGRQ), which is a standardized self-administered airway disease-specific instrument [14]. Validated translations of the standard SGRQ questionnaire featuring three domains (symptoms, activities, and impacts) were used [15]. Scores on the SGRQ range from o (no disturbance in health-related quality of life) to 100. Health status was assessed using the 36-item short-form health survey (SF36). The SF-36 contains 36 items that measure health in each of eight multi-item dimensions and explores functional status, well-being, and overall self-evaluation of health $[16,17]$. For each dimension, the item scores are coded, summed, and transformed to yield a final score ranging from o (worst health) to 100 (best health) [16]. Depression and anxiety symptoms were recorded using the hospital anxiety and depression scale (HADS) $[18,19]$, which comprises seven-item anxiety and depression subscales. The scores range from 0 to 21 for both HADS-anxiety (HADS-A) and HADS-depression (HADS-D). A score of o to 7 on either subscale is regarded as being within the normal range, and a score of $\geq 8$ indicates the probable presence of a mood disorder [20].

\section{Statistical analysis}

Continuous data are expressed as mean \pm standard de- 
viation $(\mathrm{SD})$ and categorical data as numbers with percentages. Analysis of variance and the Kruskal-Wallis test were used to compare normally and non-normally distributed continuous variables, respectively, and the chi-squared or Fisher exact test (for small numbers) were used to compare categorical variables. Comparison with $p$ values of $<0.1$ on univariate analysis were included in multivariate logistic regression analysis to identify factors associated with low-level PA. Backward elimination was performed to eliminate any predictor for which the $p$ value in the multivariate model exceeded 0.05 . All statistical analyses were performed using SPSS version 18.0 (SPSS Inc., Chicago, IL, USA), with the two-tailed significance level set at 0.05 .

\section{RESULTS}

\section{Patients characteristics and comparison of clinical data by PA status}

In total, 160 patients were included. Table 1 shows their characteristics. Of all patients, 149 (93.1\%) were male, and the mean patient age was $71.8 \pm 4.5$ years. The mean time since COPD diagnosis was 104.6 \pm 128.6 months. Of all patients, 25 lived alone and 137 were unemployed (15.6\% and $85.6 \%$, respectively). The most common comorbidities were hypertension (36 patients, 22.5\%) and diabetes (14, 8.8\%). The mean numbers of admission and emergency room visits over the previous year were $1.2 \pm$ 0.7 and $1.6 \pm 1.1$, respectively.

The mean daily walking time was $31.4 \pm 47.3$ minutes for all patients, of whom $64.4 \%$ exhibited low-level PA (<

Table 1. Characteristics according to the degree of physical activity

\begin{tabular}{|c|c|c|c|c|c|}
\hline Parameter & $\operatorname{Total}(\mathrm{n}=160)$ & Low $(n=103)$ & Moderate $(n=26)$ & $\operatorname{High}(\mathrm{n}=31)$ & $p$ value \\
\hline Age, yr & $71.8 \pm 4.5$ & $71.6 \pm 4.5$ & $71.7 \pm 4.4$ & $72.2 \pm 4.7$ & 0.833 \\
\hline Male sex & $149(93.1)$ & $93(90.3)$ & $25(96.2)$ & $31(100)$ & 0.136 \\
\hline \multicolumn{6}{|l|}{ Smoking } \\
\hline Non-smoker & $10(6.3)$ & $8(7.8)$ & $1(3.8)$ & $1(3.2)$ & 0.266 \\
\hline Former smoker & $119(74.4)$ & $79(76.7)$ & $16(61.5)$ & $24(77 \cdot 4)$ & \\
\hline Current smoker & $31(19.4)$ & $16(15 \cdot 5)$ & $9(34.6)$ & $6(19.4)$ & \\
\hline Pack-years & $43.8 \pm 28.0$ & $45.0 \pm 29.6$ & $44.0 \pm 23.1$ & $40.0 \pm 27.2$ & 0.692 \\
\hline \multicolumn{6}{|l|}{ Spirometry values } \\
\hline FVC, L & $2.8 \pm 0.9$ & $2.77 \pm 0.90$ & $2.83 \pm 0.85$ & $2.82 \pm 0.73$ & 0.920 \\
\hline $\mathrm{FEV}_{1}, \mathrm{~L}$ & $1.5 \pm 0.6$ & $1.41 \pm 0.57$ & $1.51 \pm 0.57$ & $1.63 \pm 0.51$ & 0.156 \\
\hline $\mathrm{FEV}_{1} \%$ predicted & $57.8 \pm 21.4$ & $55 \cdot 3 \pm 22.0$ & $59.6 \pm 21.9$ & $61.4 \pm 18.2$ & 0.321 \\
\hline $\mathrm{FEV}_{1} / \mathrm{FVC}$ & $53 \cdot 6 \pm 14 \cdot 3$ & $51.7 \pm 13.9$ & $54.8 \pm 15.5$ & $58.8 \pm 13 \cdot 3$ & 0.045 \\
\hline BMI, kg/m² & $21.9 \pm 3.1$ & $21.8 \pm 3 \cdot 3$ & $22.3 \pm 2.6$ & $21.9 \pm 3.0$ & 0.807 \\
\hline \multicolumn{6}{|c|}{ Dyspnea (MMRC scale) } \\
\hline $0-1$ & $111(69.4)$ & $63(61.2)$ & $22(84.6)$ & $26(83.9)$ & 0.010 \\
\hline$\geq 2$ & $49(30.6)$ & $40(38.8)$ & $4(15 \cdot 4)$ & $5(16.1)$ & \\
\hline 6MWD, m & $324.2 \pm 85.6$ & $310.6 \pm 90.5$ & $342.2 \pm 75.2$ & $354.3 \pm 66.5$ & 0.022 \\
\hline BODE index score & $3.2 \pm 2.4$ & $3.7 \pm 2.5$ & $2.6 \pm 2.0$ & $2.3 \pm 1.8$ & 0.005 \\
\hline \multicolumn{6}{|l|}{ Biomarker } \\
\hline CRP & $0.7 \pm 1.6$ & $0.6 \pm 1.1$ & $1.2 \pm 2.7$ & $0.5 \pm 1.5$ & 0.162 \\
\hline Fibrinogen & $360.9 \pm 112.2$ & $355.5 \pm 112.3$ & $382.2 \pm 103.1$ & $360.4 \pm 120.7$ & 0.561 \\
\hline
\end{tabular}

Values are presented as mean $\pm \mathrm{SD}$ or number (\%).

FVC, forced vital capacity; $\mathrm{FEV}_{1}$, forced expiratory volume in 1 second; BMI, body mass index; MMRC, modified medical research council; 6MWD, 6-minute walk distance; BODE, body mass index, degree of airway obstruction, dyspnea, and exercise capacity; CRP, C-reactive protein. 
$30 \mathrm{~min} /$ day), $16.3 \%$ moderate-level PA (30 to $60 \mathrm{~min} /$ day), and $19.4 \%$ high-level PA ( $\geq 60 \mathrm{~min} /$ day). Table 1 compares the clinical characteristics among the three groups. Upon univariate analysis, a decreased exercise capacity in the 6-minute walk test and an increased dyspnea score (MMRC $\geq 2$ ) were significantly associated with low-level PA $(p<0.1)$. The severity of COPD (derived using the BODE [body mass index, degree of airway obstruction, dyspnea, and exercise capacity] index) was inversely related to PA. Indices of lung function, such as forced expiratory volume in 1 second $\left(\mathrm{FEV}_{1}\right)$, did not differ significantly among the three groups.

\section{Questionnaire scores}

Table 2 shows the relationships of PA with HRQL, health status, and psychosocial factors. Patients with low-levels of PA tended to have a reduced HRQL (high total SGRQ scores) and poor health (low SF-36 scores). Additionally, the proportions of patients with anxiety (HADS-A score of $\geq 8$ ) and depression (HADS-D score of $\geq 8$ ) were higher in patients with low-level PA.

\section{Predictors of low-level PA in elderly patients}

We used univariate and multivariate logistic regression models to identify predictors of low-level PA in elderly patients with COPD. Univariate analysis indicated that six factors (MMRC score of $\geq 2$, 6-minute walk test result of $<250 \mathrm{~m}$, total SGRQ score, SF-36 physical functioning score, HADS-D score of $\geq 8$, and HADS-A score of $\geq 8$ ) should be included in the multivariate model (Table 3). The factors independently associated with low-level PA on multivariate analysis were severe dyspnea (MMRC score of $\geq 2$ ) and depression (HADS-D score of $\geq 8$ ) (Table 4).

\section{DISCUSSION}

We explored the clinical factors associated with low-level PA in elderly patients with COPD. The median age of all patients was 71.8 years, and two-thirds reported low-levels of PA. This proportion is higher than those of previous studies that included middle-aged patients [11].

Table 2. Comparison of questionnaire scores according to the physical activity

\begin{tabular}{|c|c|c|c|c|c|}
\hline Parameter & Total $(n=160)$ & Low $(\mathrm{n}=103)$ & Moderate $(n=26)$ & $\operatorname{High}(n=31)$ & $p$ value \\
\hline \multicolumn{6}{|l|}{ SGRQ } \\
\hline Symptoms domain & $35.7 \pm 21.2$ & $36.7 \pm 22.3$ & $37.4 \pm 16.9$ & $31.1 \pm 20.9$ & 0.400 \\
\hline Activity domain & $57.1 \pm 25 \cdot 3$ & $61.1 \pm 24.1$ & $56.5 \pm 26.3$ & $44 \cdot 3 \pm 24.8$ & 0.005 \\
\hline Impact domain & $28.7 \pm 21.7$ & $32.2 \pm 22.0$ & $26.0 \pm 24.5$ & $19.5 \pm 14.8$ & 0.013 \\
\hline Total score & $38.5 \pm 20.1$ & $41.7 \pm 20.0$ & $37.2 \pm 21.4$ & $28.9 \pm 16.6$ & 0.007 \\
\hline \multicolumn{6}{|l|}{$\mathrm{SF}-36$} \\
\hline Physical functioning & $60.4 \pm 26.3$ & $55 \cdot 3 \pm 26.7$ & $62.5 \pm 27.8$ & $75.7 \pm 16.7$ & 0.001 \\
\hline Social functioning & $89 \cdot 3 \pm 21.3$ & $86.0 \pm 24.4$ & $93.8 \pm 15.5$ & $96.4 \pm 9.2$ & 0.030 \\
\hline Role physical & $53 \cdot 9 \pm 45 \cdot 5$ & $45.6 \pm 44.8$ & $54.8 \pm 45 \cdot 3$ & $80.7 \pm 38.6$ & 0.001 \\
\hline Role emotional & $77 \cdot 9 \pm 39 \cdot 7$ & $71.5 \pm 43.1$ & $82.1 \pm 38.0$ & $95.7 \pm 18.7$ & 0.009 \\
\hline Mental health & $66.7 \pm 22.9$ & $64.5 \pm 21.9$ & $70.9 \pm 24.1$ & $70.7 \pm 24.5$ & 0.245 \\
\hline Vitality (energy/fatigue) & $39.4 \pm 16.4$ & $36.9 \pm 15.9$ & $40.6 \pm 15.0$ & $46.6 \pm 17.4$ & 0.013 \\
\hline Pain & $85.7 \pm 25.0$ & $83.2 \pm 26.5$ & $89.4 \pm 21.4$ & $90.9 \pm 21.8$ & 0.230 \\
\hline General health & $47 \cdot 4 \pm 24 \cdot 3$ & $45.1 \pm 23.9$ & $44 \cdot 4 \pm 24 \cdot 7$ & $57.6 \pm 23.5$ & 0.033 \\
\hline HADS-A & $3.9 \pm 4.1$ & $4 \cdot 4 \pm 4 \cdot 3$ & $3 \cdot 5 \pm 4 \cdot 4$ & $2.6 \pm 2.8$ & 0.090 \\
\hline HADS-D & $6.9 \pm 4.1$ & $7 \cdot 7 \pm 4 \cdot 3$ & $6.2 \pm 3.8$ & $5.0 \pm 3.2$ & 0.005 \\
\hline $\mathrm{HADS}-\mathrm{A} \geq 8$ & $26(16.3)$ & $21(20.4)$ & $4(15 \cdot 4)$ & $1(3.2)$ & 0.059 \\
\hline $\mathrm{HADS}-\mathrm{D} \geq 8$ & $69(43.1)$ & $5^{2}(50.5)$ & $8(30.8)$ & $9(29.0)$ & 0.042 \\
\hline
\end{tabular}

Values are presented as mean $\pm \mathrm{SD}$ or number (\%).

SGRQ, St. George's respiratory questionnaire; SF-36, medical outcomes short form-36; HADS-A, hospital anxiety and depression scale-anxiety; HADS-D, hospital anxiety and depression scale-depression. 
Table 3. Factors associated with low degree of physical activity by univariate analysis

\begin{tabular}{lcc}
\hline Factor & HR $(95 \%$ CI $)$ & p value \\
\hline MMRC scale $(\geq 2)$ & $3.386(1.499-7.648)$ & 0.003 \\
6MWD $(<250 \mathrm{~m})$ & $4.474(1.476-13.566)$ & 0.008 \\
SGRQ total score & $1.024(1.006-1.043)$ & 0.008 \\
SF-36 physical functioning & $0.977(0.964-0.991)$ & 0.001 \\
HADS-A $\geq 8$ & $2.663(0.946-7.500)$ & 0.064 \\
HADS-D $\geq 8$ & $2.399(1.208-4.766)$ & 0.012 \\
\hline
\end{tabular}

$\mathrm{HR}$, hazard ratio; CI, confidence interval; MMRC, modified medical research council; 6MWD, 6-minute walk distance; SGRQ, St. George's respiratory questionnaire; SF-36, medical outcomes short form-36; HADS-A, hospital anxiety and depression scale-anxiety; HADS-D, hospital anxiety and depression scale-depression.

Table 4. Factors associated with low degree of physical activity by multivariate analysis

\begin{tabular}{lcc}
\hline Factor & HR $(95 \% \mathrm{CI})$ & $p$ value \\
\hline MMRC scale $(\geq 2)$ & $2.550(1.075-6.049)$ & 0.034 \\
6MWD $(<250 \mathrm{~m})$ & $2.697(0.832-8.741)$ & 0.098 \\
HADS-D $\geq 8$ & $2.076(1.016-4.241)$ & 0.045 \\
\hline
\end{tabular}

HR, hazard ratio; CI, confidence interval; MMRC, modified medical research council; 6MWD, 6-minute walk distance; HADS-D, hospital anxiety and depression scale-depression.

Our principal findings were that dyspnea severity and depression were independently associated with low-level PA in elderly patients with COPD. Although several studies have sought to identify predictors of low-level PA in patients with COPD $[8,10,11]$, few have specifically examined clinical factors influencing PA in elderly patients. Therefore, this is the first study to analyze PA patterns and predictors of low-level PA in elderly patients with COPD.

Of all patients, 30.6\% had severe dyspnea (MMRC score of $\geq 2$ ), which was independently associated with low-level PA. The etiology of dyspnea is multifactorial, but recent mechanistic studies suggest that dynamic lung hyperinflation-induced volume restriction with consequent neuromechanical uncoupling of the respiratory system are associated with exertional dyspnea and exercise intolerance in patients with COPD [21]. Previous studies found that relief of exertional dyspnea and improved exercise endurance following prescription of bronchodilator therapy were correlated with reduced lung hyperinflation [21]. In addition, dyspnea was the only symptom predicting variation in functional performance, and oxygen may influence such performance and the dyspnea symptoms per se in patients with COPD [22]. Although COPD treatments have traditionally sought to improve both pathological and physiological parameters, assessment of the response to treatment may be key when it is sought to predict improvements in the daily activities and outcomes of elderly patients.

Depression is more prevalent in patients with COPD than in those with other medical conditions [23]. Although the true prevalence of depression in elderly patients with COPD is unclear, several studies have reported that the level was high $(>40 \%)[24,25]$. In our study, the prevalence was $43.1 \%$, which is similar to that of previous studies. We assessed clinical depression using the HADS, which is a valid and reliable self-rating scale measuring anxiety and depression in both hospital and community settings [19]. In the original study, the cut-off scores for possible and probable depression were 7/8 and 10/11, respectively [19]. In our study, possible (HADS-D score of 8 to 10) and probable (HADS-D score of $\geq 11$ ) depression were evident in 36 patients (22.5\%) and 33 (20.6\%), respectively. We used a score of 8 as the depression threshold because possible or subthreshold depression is also known to be an independent predictor of physical disability and impaired HRQL [25]. In most studies, the optimal balance between sensitivity and specificity was evident when caseness was defined by a score of $\geq 8$ on the HADS-D.

Unfortunately, little is known about the mechanism that depression develops in elderly patients with COPD. Very few studies have explored structural brain pathology or cognitive dysfunction specifically in such patients. Magnetic resonance imaging-based quantitative assessment of brain pathology affords direct evidence of psychological and mood changes [26]. Age-related diseasespecific processes, including hypoxia and inflammatory changes, compromise the integrity of the frontostriatal pathways, amygdala, and hippocampus, increasing the vulnerability of elderly patients with COPD to depression [27]. In our study, depression was independently associated with low-level of PA. Because this study is the cross-sectional study, it does not allow establishment of 
causal relationship. However, previous studies reported that disease-specific fears contributed to disability and avoidance of PA in subjects with COPD [28]. In addition, a recent study reported that symptoms of depression were prospectively associated with a reduction in PA 6 months later in COPD patients [29]. Therefore we suggest that the presence of depression could decrease the level of PA. However, no direct association has been yet identified. Further studies are needed.

In our present study, only one of the 69 patients with depression was actually treated for the condition. Undetected and untreated depression reduces compliance with medical treatment, quality-of-life, and overall survival of the elderly $[30,31]$. Therefore, appropriate screening tools and therapy are required to reduce depressive symptoms and the associated disability in elderly patients with COPD [32]. Treatment of the underlying COPD is of course important, but this is often not enough to achieve remission of depression. Antidepressants, psychotherapy, or both are preferred treatment options for late-life major depression [33]. However, aging influences drug pharmacodynamic responses differently at the various pharmacokinetic stages. When prescribing for elderly patients, careful and specific assessment of drug-related problems is required [34]. In patients with minor depression, nonspecific supportive interventions are acceptable alternatives.

In the present study, we found no significant association between lung function and PA. In several studies that included middle-aged patients with COPD, the $\mathrm{FEV}_{1}$ and the maximal voluntary ventilation exhibited weak to moderate positive associations with PA [12]. However, only a few studies have explored the relationships between PA and measures of lung function. In elderly patients with COPD, lung function may not correlate with low-level PA.

Our study had several limitations. First, the number of the patients is relatively small in our study. Second, we did not consider clinical differences among elderly patients; for example, we did not subdivide patients into those aged $>80$ and 65 to 80 years. Furthermore, the $\mathrm{FEV}_{1} /$ forced vital capacity (FVC) ratio declines with age, and the use of a fixed $\mathrm{FEV}_{1} / \mathrm{FVC}$ ratio of 0.7 as the threshold for airway obstruction in the elderly remains controversial. Third, we could not assess etiologic or causal links between the level of PA and dyspnea or de- pression because of the cross-sectional nature of this analysis. Finally, we used self-reported questionnaire rather than a direct measures, such as accelerometers, to establish the PA level. Although objective measures can provide more accurate way, they are not feasible in routine practice. Several studies have demonstrated the correlation of self-reported walking times per day (patients who walked $<30,30$ to 60 , and $\geq 60$ minutes) with health-related quality of life and functional status [11].

In conclusion, two-thirds of elderly patients with COPD reported low-level PA. Severe dyspnea and depression were independently associated with this lowlevel of PA. Further work with larger populations would afford more insight into predictors of low-level PA in elderly patients with COPD.

\section{KEY MESSAGE}

1. Severe dyspnea and depression are independent risk factor for low-level physical activity (PA) in elderly patients with chronic obstructive pulmonary disease (COPD). Obviously, these clinical factors alone do not explain why the PA level was low. Nevertheless, assessment of dyspnea and symptom control may be the key to increase daily activity in elderly patients with COPD.

2. The application of adequate screening for presence of depression and aggressive treatment for depression could improve the level of PA.

\section{Conflict of interest}

No potential conflict of interest relevant to this article was reported.

\section{Acknowledgments}

This research was supported by the Basic Science Research Program the National Research Foundation of Korea (NRF) funded by the Ministry of Education, Science and Technology (2009-0088833).

\section{REFERENCES}

1. Rennemark M, Lindwall M, Halling A, Berglund J. Relationships between physical activity and perceived qual- 
ities of life in old age: results of the SNAC study. Aging Ment Health 2009;13:1-8.

2. Aalami OO, Fang TD, Song HM, Nacamuli RP. Physiological features of aging persons. Arch Surg 2003;138:10681076.

3. Franceschi C, Capri M, Monti D, et al. Inflammaging and anti-inflammaging: a systemic perspective on aging and longevity emerged from studies in humans. Mech Ageing Dev 2007;128:92-105.

4. Lopez AD, Shibuya K, Rao C, et al. Chronic obstructive pulmonary disease: current burden and future projections. Eur Respir J 2006;27:397-412.

5. Belman MJ. Exercise in patients with chronic obstructive pulmonary disease. Thorax 1993;48:936-946.

6. Yohannes AM, Roomi J, Waters K, Connolly MJ. Quality of life in elderly patients with COPD: measurement and predictive factors. Respir Med 1998;92:1231-1236.

7. Gosker HR, Lencer NH, Franssen FM, van der Vusse GJ, Wouters EF, Schols AM. Striking similarities in systemic factors contributing to decreased exercise capacity in patients with severe chronic heart failure or COPD. Chest 2003;123:1416-1424.

8. Garcia-Aymerich J, Serra I, Gomez FP, et al. Physical activity and clinical and functional status in COPD. Chest 2009;136:62-70.

9. Garcia-Aymerich J, Lange P, Benet M, Schnohr P, Anto JM. Regular physical activity reduces hospital admission and mortality in chronic obstructive pulmonary disease: a population based cohort study. Thorax 2006;61:772-778.

10. Garcia-Aymerich J, Felez MA, Escarrabill J, et al. Physical activity and its determinants in severe chronic obstructive pulmonary disease. Med Sci Sports Exerc 2004;36:16671673 .

11. Miravitlles M, Cantoni J, Naberan K. Factors associated with a low level of physical activity in patients with chronic obstructive pulmonary disease. Lung 2014;192:259-265.

12. Watz H, Pitta F, Rochester CL, et al. An official European Respiratory Society statement on physical activity in COPD. Eur Respir J 2014;44:1521-1537.

13. Kim KU, Park HK, Jung HY, et al. Association of depression with disease severity in patients with chronic obstructive pulmonary disease. Lung 2014;192:243-249.

14. Jones PW, Quirk FH, Baveystock CM. The St George's respiratory questionnaire. Respir Med 1991;85 Suppl B:25-31.

15. Kim YS, Byun MK, Jung WY, et al. Validation of the Korean version of the St. George's respiratory questionnaire for patients with chronic respiratory disease. Tuberc Respir Dis 2006;61:121-128.

16. Brazier JE, Harper R, Jones NM, et al. Validating the SF36 health survey questionnaire: new outcome measure for primary care. BMJ 1992;305:160-164.

17. Ware JE Jr, Sherbourne CD. The MOS 36-item short-form health survey (SF-36). I. Conceptual framework and item selection. Med Care 1992;30:473-483.

18. Oh SM, Min KJ, Park DB. A study on the standardization of the hospital anxiety and depression scale for Koreans: a comparison of normal, depressed and anxious groups. J Korean Neuropsychiatr Assoc 1999;38:289-296.

19. Zigmond AS, Snaith RP. The hospital anxiety and depression scale. Acta Psychiatr Scand 1983;67:361-370.

20. Bjelland I, Dahl AA, Haug TT, Neckelmann D. The validity of the hospital anxiety and depression scale: an updated literature review. J Psychosom Res 2002;52:69-77.

21. O'Donnell DE, Laveneziana P. Dyspnea and activity limitation in COPD: mechanical factors. COPD 2007;4:225236.

22. Reishtein JL. Relationship between symptoms and functional performance in COPD. Res Nurs Health 2005;28:3947.

23. Light RW, Merrill EJ, Despars JA, Gordon GH, Mutalipassi LR. Prevalence of depression and anxiety in patients with COPD: relationship to functional capacity. Chest 1985;87:35-38.

24. Yohannes AM, Baldwin RC, Connolly MJ. Depression and anxiety in elderly outpatients with chronic obstructive pulmonary disease: prevalence, and validation of the BASDEC screening questionnaire. Int J Geriatr Psychiatry 2000;15:1090-1096.

25. Yohannes AM, Baldwin RC, Connolly MJ. Prevalence of sub-threshold depression in elderly patients with chronic obstructive pulmonary disease. Int J Geriatr Psychiatry 2003;18:412-416.

26. Zhang H, Wang X, Lin J, et al. Grey and white matter abnormalities in chronic obstructive pulmonary disease: a case-control study. BMJ Open 2012;2:e000844.

27. Alexopoulos GS. Depression in the elderly. Lancet 2005; 365:1961-1970.

28. Keil DC, Stenzel NM, Kuhl K, et al. The impact of chronic obstructive pulmonary disease-related fears on disease-specific disability. Chron Respir Dis 2014;11:31-40.

29. Duenas-Espin I, Demeyer H, Gimeno-Santos E, et al. Depression symptoms reduce physical activity in COPD 
patients: a prospective multicenter study. Int J Chron Obstruct Pulmon Dis 2016;11:1287-1295.

30. Bosley CM, Corden ZM, Rees PJ, Cochrane GM. Psychological factors associated with use of home nebulized therapy for COPD. Eur Respir J 1996;9:2346-2350.

31. Pulska T, Pahkala K, Laippala P, Kivela SL. Follow up study of longstanding depression as predictor of mortality in elderly people living in the community. BMJ 1999;318:432-433.

32. Bartels SJ, Dums AR, Oxman TE, et al. Evidence-based practices in geriatric mental health care: an overview of systematic reviews and meta-analyses. Psychiatr Clin North Am 2003;26:971-990.

33. Alexopoulos GS, Katz IR, Reynolds CF 3rd, Carpenter D, Docherty JP; Expert Consensus Panel for Pharmacotherapy of Depressive Disorders in Older Patients. The expert consensus guideline series: pharmacotherapy of depressive disorders in older patients. Postgrad Med 2001;Spec No Pharmacotherapy:1-86.

34. Hilmer SN, McLachlan AJ, Le Couteur DG. Clinical pharmacology in the geriatric patient. Fundam Clin Pharmacol 2007;21:217-230. 